\title{
Comparison of three digestive tract reconstruction methods for the treatment of Siewert II and III adenocarcinoma of esophagogastric junction: a prospective, randomized controlled study
}

\author{
Zhiguo Li, Jianhong Dong*, Qingxing Huang, Wanhong Zhang and Kai Tao
}

\begin{abstract}
Background: The incidence of adenocarcinoma of esophagogastric junction (AEG) has recently risen worldwide, including in Eastern Asia. The aim of the study was to explore the short-term and long-term clinical efficacy of piggyback jejunal interposition reconstruction single-tract reconstruction (PJIRSTR), piggyback jejunal interposition reconstruction double-tract reconstruction (PJIRDTR), and total gastrectomy esophageal jejunal Roux-en-Y anastomosis (TGRY) for the treatment of Siewert II and III AEG patients.
\end{abstract}

Methods: A total of 300 Siewert II and III AEG patients admitted to Shanxi Tumor Hospital from June 2015 to December 2017 were prospectively selected. Patients were randomly divided into PJIRSTR group $(n=98)$, PJIRDTR group ( $n=103)$, and TGRY group $(n=99)$ using the random number table method.

Results: There were no statistically significant differences in total operation time, intraoperative blood loss, time of first anal exhaust, and postoperative hospital stay among the three groups $(F=2.526,0.457,0.234,0.453 ; P>0.05)$. The reconstruction time of PJIRSTR group and PJIRDTR group was longer than that of TGRY group $(P<0.01)$. There were no significant differences in cases of anastomotic leakage, anastomotic bleeding, abdominal infection, incision infection, ileus, and dumping syndrome in three groups $(P>0.05)$. The incidence of reflux esophagitis at $3,6,12$, and 18 months after surgery in the PJIRSTR group and the PJIRDTR group were significantly lower than TGRY group in the same period $(P<0.05)$. Compared with PJIRDTR group and TGRY group, PJIRSTR group had a small fluctuation range of postoperative nutrition indexes and had basically recovered to the preoperative level at 18 months. Four patients of Visick grade IV presented in TGRY group 18 months postoperatively, which was significantly higher compared with the other two groups.

Conclusion: Compared with PJIRDTR and TGRY, PJIRSTR can significantly reduce the incidence of postoperative reflux esophagitis and improve the long-term nutritional status of patients.

Trial registration: Chinese Clinical Trial Registry, ChiCTR-IIR-16007733. Registered 07 November 2015 Retrospectively registered, http://www.chictr.org.cn/searchproj.aspx.

Keywords: Piggyback jejunal interposition reconstruction single-tract reconstruction, Piggyback jejunal interposition reconstruction double-tract reconstruction, Total gastrectomy esophageal jejunal Roux-en-Y anastomosis, Reflux esophagitis

\footnotetext{
* Correspondence: asdjh666@163.com

Department of Minimal Invasive Digestive Surgery, Shanxi Tumor Hospital,

Shanxi Medical University, Taiyuan 030013, China
}

(c) The Author(s). 2019 Open Access This article is distributed under the terms of the Creative Commons Attribution 4.0 International License (http://creativecommons.org/licenses/by/4.0/), which permits unrestricted use, distribution, and reproduction in any medium, provided you give appropriate credit to the original author(s) and the source, provide a link to the Creative Commons license, and indicate if changes were made. The Creative Commons Public Domain Dedication waiver (http://creativecommons.org/publicdomain/zero/1.0/) applies to the data made available in this article, unless otherwise stated. 


\section{Background}

Adenocarcinoma of esophagogastric junction (AEG) refers to the adenocarcinoma that occurs in the esophagogastric junction and within the range of $5 \mathrm{~cm}$ in both directions. In recent years, the incidence of AEG in eastern and western countries is increasing year by year [1].

At present, there is still a great controversy about the methods of digestive tract reconstruction for the treatment of AEG. European and American scholars believe that total gastrectomy (TG) should be selected since proximal gastrectomy (PG) may lead to the recurrence of gastric cancer due to cardiac loss, high incidence of long-term complications, and incomplete lymph node dissection [2]. However, Japanese scholars believe that PG should be selected because retention of residual stomach not only stores part of food and water but also ensures the absorption of nutrients such as iron, thus reducing the incidence of anemia and other complications [3]. With more and more in-depth studies on AEG, the views of Italian scholars have been accepted by the vast majority of scholars, who believe that there is no significant correlation between the survival rate of AEG patients and whether they receive PG or TG [4].

Proximal gastrectomy combined with jejunal interposition reconstruction has become an ideal choice for the treatment of for Siewert II and III AEG, since studies have shown that it can ensure the surgical safety, achieve an ideal radical cure, and achieve comparable 5-year overall survival with TG [5-7]. In China, piggyback jejunal interposition reconstruction (PJIR), proposed by Shanxi Tumor Hospital, was performed on the basis of Roux-en-Y jejunal anastomosis on the jejunum of the esophagus, and then jejunal remnant jejunostomy was performed on the appropriate location of jejunum loop. PJIR is divided into single-tract reconstruction (STR) and double-tract reconstruction (DTR), and its efficacy has been preliminarily verified [8]. However, there is still no consensus on the method of digestive tract reconstruction for Siewert II and III AEG patients.

This study prospectively analyzed the clinical pathological data of 300 Siewert II and III AEG patients and discussed the clinical effect of piggyback jejunal interposition reconstruction single-tract reconstruction (PJIRSTR), piggyback jejunal interposition reconstruction double-tract reconstruction (PJIRDTR), and total gastrectomy esophageal jejunal Roux-en-Y anastomosis (TGRY) for the treatment for AEG.

\section{Methods}

\section{Design}

We conducted a single-center, prospective, interventional, randomized therapeutic clinical trial to discuss the clinical effect of PJIRSTR, PJIRDTR, and TGRY for the treatment for AEG. This study was approved by the ethics committee of Shanxi Tumor Hospital. All patients signed informed consent and all surgeries were performed by the same surgeon. The study was registered at Chinese Clinical Trial Registry (http://www.chictr.org. cn/index.aspx; no. ChiCTR-IIR-16007733).

\section{Participants}

About 300 patients with AEG admitted to Shanxi Tumor Hospital from June 2015 to December 2017 were selected. Inclusion criteria: Patients (1) aged 18 to 75 years; (2) with $\mathrm{cT}_{1-3} \mathrm{~N}_{0} \mathrm{M}_{0}$ [TNM staging (AJCC 8th edition [8]) was used as the standard Siewert II or III type AEG]. The location of the AEG was defined as lower margin of palisading small vessels on endoscopy according to the Japanese Classification of Esophageal Cancer (11th edition) [9]; (3) tumor from lower dentate line $\leq 4 \mathrm{~cm}$, the tumor diameter $<4 \mathrm{~cm}$; (4) with primary tumors without distant metastasis; (5) with no surgical contraindications; and (6) with no history of malignant tumor surgery. Exclusion criteria: Patients (1) with other serious diseases that would not tolerate anesthesia and surgery, (2) had locally advanced or invasive carcinoma and (3) aged > 75 years old. About 300 patients were divided into PJIRSTR group $(n=98)$, PJIRDTR group $(n=103)$, and TGRY group $(n=99)$ according to the random number table.

\section{Surgical operation}

Within 2 weeks after enrolment, surgeons performed surgery via the abdominal transhiatal (TH) approach, according to the study protocol. The upper abdominal midline incision was used, and $\mathrm{D}^{+}$or $\mathrm{D} 2$ lymph node dissection was performed. During the operation, the proximal and distal incisional margins of the resected specimens with suspicious positive incisional margins were sent for rapid pathology, and then the digestive tract reconstruction was performed.

For PJIRSTR group, jejunum was cut off about 20-25 $\mathrm{cm}$ from the Treitz ligament and was then lifted up to the end of the esophagus before or after the colon, followed by esophageal and jejunal anastomosis using 26\# stapler. Lateral anastomosis was performed between the jejunum and the posterior wall of the remnant stomach about $12 \sim 15 \mathrm{~cm}$ from the esophageal jejunal anastomosis. The jejunum was closed with a closure device at $3 \mathrm{~cm}$ below the anastomosis to completely block the jejunum content passage. The jejunum was anastomosed with the proximal jejunum at the distal end of the ligation about $5 \sim 10 \mathrm{~cm}$, as shown in Fig. 1. For PJIRDTR group, jejunum was not closed, but the distance between the gastrojejunal anastomosis and jejuno-jejunal anastomosis should be more than $30 \mathrm{~cm}$ to prevent reflux. The remaining surgical methods were the same as PJIRSTR group (Fig. 2). For TGRY group, the duodenal stump 


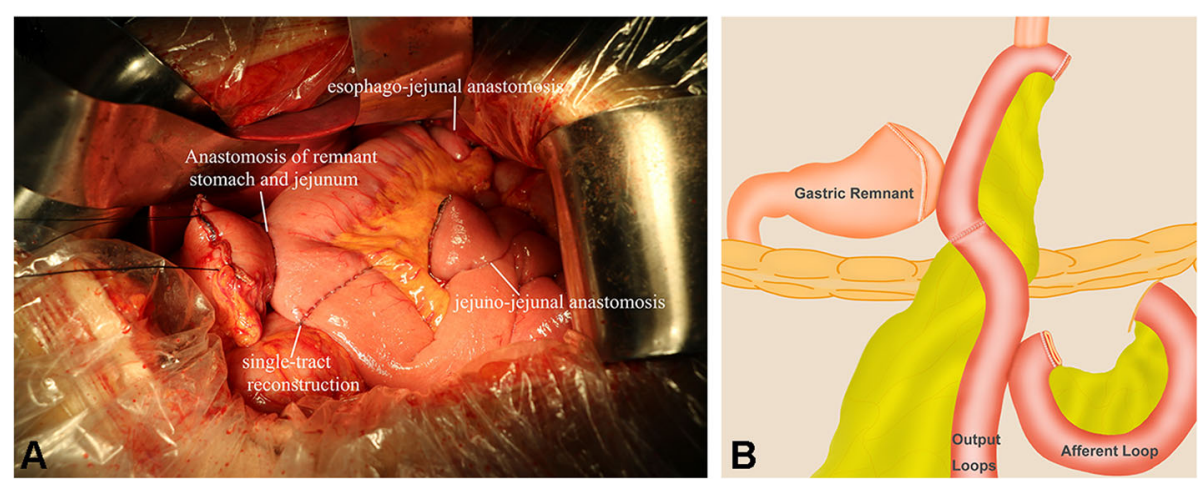

Fig. 1 The piggyback jejunal interposition reconstruction single-tract reconstruction. $\mathbf{a}$ is the picture of the single-tract reconstruction of proximal gastrectomy with piggyback jejunal interposition reconstruction; (b) is the schematic diagram

was closed, jejunum was cut off $20 \mathrm{~cm}$ from the distal end of the Treitz ligament, and the distal jejunum was anastomosed with the lower end of the esophagus. Meanwhile, proximal jejunum and distal jejunum were anastomosed about 40-45 cm from the distal end of the jejunal esophageal anastomosis. Three groups of patients were indwelling nutrition tube, not indwelling gastric tube. From the first day after operation, enteral nutrition support was performed with nutrient tube for 1 week, and $400 \mathrm{ml}$ Nestle Healthcare Nutrition NUTREN OPTIMUM was infused daily; after 1 week, patients began to take oral nutrition supplement (Nestle Healthcare Nutrition NUTREN OPTIMUM, 1500 ml, daily).

\section{Observation indexes and Evaluation criteria}

Operation time, digestive tract reconstruction time, intraoperative blood loss, first anal exhaust time, and postoperative hospital stay were recorded. Postoperative complications including anastomotic leakage, anastomotic bleeding, abdominal infection, incision infection, ileus, and dumping syndrome were observed. The postoperative pathological examinations showed that patients with positive lymph nodes or tumor invading all layers of gastric wall underwent the six-cycle SOX chemotherapy.

\section{Postoperative nutritional status and endoscopic gastroesophageal reflux}

All the patients in three groups were followed up to 30 months after surgery, and the patients were followed up by telephone and returned to the hospital regularly for review. Visick score [8] was used to evaluate gastroesophageal reflux symptoms 18 months postoperatively: Visick grade I, asymptomatic; Visick grade II, occasional symptoms; Visick grade III, apparent but tolerable symptoms; and Visick grade IV, apparent and intolerable symptoms.

Nutritionist, specialist nurses, and physicians were involved, and nutritional adjustment and postoperative guidance were performed during follow-up of patients. Patients included were given medical cost reduction during follow-up; therefore the compliance is good. Body weight and the nutritional status including hemoglobin, total serum protein, serum albumin, and blood vitamin
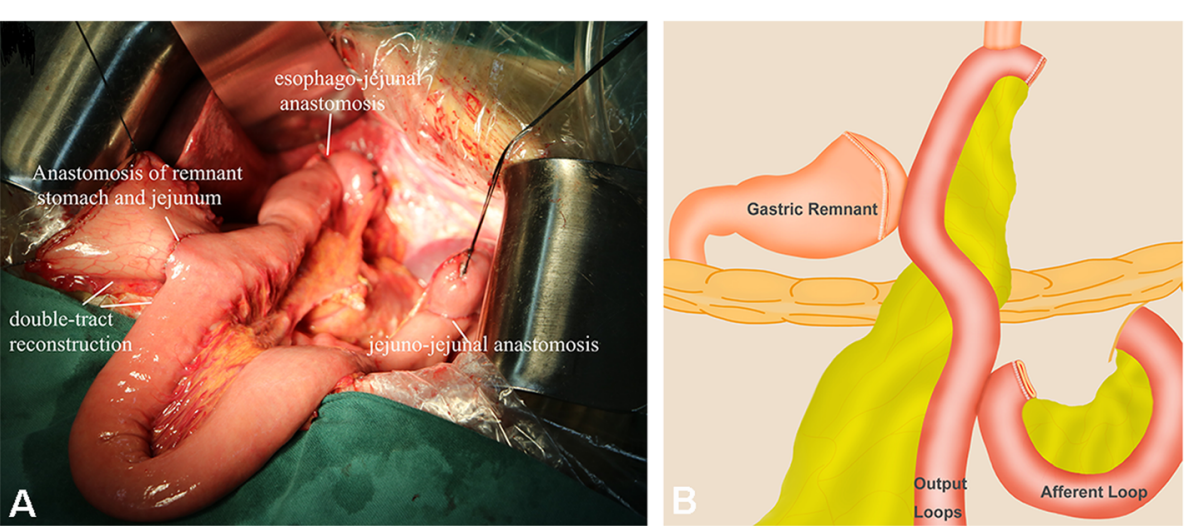

Fig. 2 The piggyback jejunal interposition reconstruction double-tract reconstruction. $\mathbf{a}$ is the picture of the double-tract reconstruction of proximal gastrectomy with piggyback jejunal interposition reconstruction; (b) is the schematic diagram 
B12 were accessed at preoperative and postoperative 3, 6, 12, and 18 months. Esophagitis was assessed using fiber-optic endoscopy, and the incidence of reflux esophagitis was observed at postoperative 3, 6, 12, and 18 months. All patients in the three groups underwent postoperative endoscopy at $3,6,12$, and 18 months to evaluate gastroesophageal reflux.

\section{Statistical methods}

Statistical analysis was performed using SPSS 19.0 software, and values are presented as mean \pm standard deviation (SD) or numbers (percentage). Between-group comparisons were analyzed by one-way ANOVA for normally distributed data. Categorical data were analyzed with the Chi-square test. $P<0.05$ was considered statistically significant.

\section{Results}

\section{Patient characteristics}

Baseline characteristics of treatment groups were well balanced (Table 1) $(P>0.05)$.

\section{Surgical outcomes}

No significant differences were observed in operation time, intraoperative blood loss, first anal exhaust time, and postoperative hospital stay among the three groups $(P>0.05)$. No patient needed combined organ resection among the three groups. There were significantly fewer

Table 1 General information

\begin{tabular}{|c|c|c|c|c|}
\hline Variables $(n, \%)$ & $\begin{array}{l}\text { PJIRSTR } \\
(n=98)\end{array}$ & $\begin{array}{l}\text { PJIRDTR } \\
(n=103)\end{array}$ & $\begin{array}{l}\text { TGRY } \\
(n=99)\end{array}$ & $P$ value \\
\hline Male & $88(89.80)$ & $90(87.38)$ & $82(82.83)$ & 0.345 \\
\hline Age (years) & & & & 0.458 \\
\hline$<60$ & $25(25.51)$ & $23(22.33)$ & $18(18.18)$ & \\
\hline$\geq 60$ & $73(74.49)$ & $80(77.67)$ & $18(18.18)$ & \\
\hline Differentiation & & & & 0.572 \\
\hline High-middle & $60(61.22)$ & $70(67.96)$ & $66(66.67)$ & \\
\hline Low & $38(38.78)$ & $33(32.04)$ & 33 (33.33) & \\
\hline TNM stage & & & & 0.562 \\
\hline I & $81(82.65)$ & $87(84.47)$ & 78 (78.79) & \\
\hline$\|$ & $17(17.35)$ & 16 (15.53) & $21(21.21)$ & \\
\hline Siewert type & & & & 0.628 \\
\hline$\|$ & $45(45.92)$ & $48(46.60)$ & $40(40.40)$ & \\
\hline III & $53(54.08)$ & $55(53.40)$ & $59(59.60)$ & \\
\hline Clinical stage & & & & 0.562 \\
\hline T1NOMO stage IA & $61(62.24)$ & $65(63.11)$ & $63(63.64)$ & \\
\hline T2NOMO stage IB & $20(20.41)$ & $22(21.36)$ & 15 (15.15) & \\
\hline T3NOM0 stage II & $17(17.35)$ & $16(15.53)$ & $21(21.21)$ & \\
\hline
\end{tabular}

lymph nodes in the PJIRSTR and PJIRDTR than in the TGRY, whereas R0 resection was performed in all patients. As shown in Table 2, the reconstruction time of PJIRSTR group and PJIRDTR group was longer than that of TGRY group $(P<0.01)$ since piggyback jejunal interposition reconstruction (PJIRSTR group and PJIRDTR group) involves the trimming of the residual stomach and anastomosis of the residual stomach and jejunum. No significant differences were noted in the early complication rates regarding anastomotic leakage, anastomotic bleeding, abdominal infection, incision infection, ileus, and dumping syndrome among three groups $(P>0.05)$ (Table 3$)$. Among them, two patients with anastomotic leakage were fully drained through the abdominal drainage tube and recovered after enteral and parenteral nutrition support. One patient with abdominal infection and two patients with incision infection complicated with incision fat liquefaction were treated by subcutaneous drainage and recovered. One case of intestinal obstruction after total gastrectomy was treated with internal conservative therapy, which was ineffective. Thus a second operation was performed and recovered. Of 300 patients, 10 underwent 6-cycle SOX chemotherapy, including 7 with perigastric lymph node metastasis and 3 with tumor invading all layers of gastric wall. In the PJIRSTR group, none of the patients developed cancer recurrence, but one patient died of acute pulmonary infarction. In the PJIRDTR group, one patient died of pneumonia without gastric cancer recurrence. In the TGRY group, one patient with pathological T4aN1M0 stage IIIA developed retroperitoneal lymph node metastasis but is currently alive.

\section{Postoperative nutritional status and reflux esophagitis} The incidence of reflux esophagitis at 3, 6, 12, and 18 months after surgery in the PJIRSTR group and the PJIRDTR group was significantly lower than TGRY group in the same period $(P<0.05)$ (Table 4$)$. Patients with reflux esophagitis in PJIRSTR group and PJIRDTR group disappeared after oral administration of esomeprazole enteric-coated tablets (AstraZeneca) and encouragement of eating solid food and patients with alkaline reflux esophagitis in TGRY group were relieved after eating solid food and avoiding lying down.

As shown in Table 5, there were no significant differences in patients of Visick grade I, Visick grade II, and Visick grade III in three groups $(P>0.05)$. There were four patients of grade IV Visick in TGRY group. After oral administration of mosapride citrate tablets (Jiangsu howson), two patients had remission of symptoms, while two patients had no remission thus had to be injected nutrition for a long time. The results of gastroscopy at postoperative 18 months were shown in Fig. 3. 
Table 2 Comparison of intraoperative and postoperative conditions among three groups

\begin{tabular}{|c|c|c|c|c|}
\hline & $\begin{array}{l}\text { PJIRSTR } \\
(n=98) \\
\end{array}$ & $\begin{array}{l}\text { PJIRDTR } \\
(n=103) \\
\end{array}$ & $\begin{array}{l}\text { TGRY } \\
(n=99) \\
\end{array}$ & $P$ value \\
\hline Lymph node dissection $(n, \%)$ & & & & 0.017 \\
\hline $\mathrm{D} 1+$ & 98 & 103 & 95 & \\
\hline D2 & 0 & 0 & 4 & \\
\hline Combined resection $(n, \%)$ & & & & - \\
\hline Gall bladder & 0 & 0 & 0 & \\
\hline Spleen & 0 & 0 & 0 & \\
\hline Lymph node dissection number & $22 \pm 3$ & $23 \pm 3$ & $37 \pm 4$ & $<0.001$ \\
\hline R0 resection $(n, \%)$ & $98(100)$ & $103(100)$ & $99(100)$ & - \\
\hline Operation time (min) & $144.37 \pm 2.51$ & $143.62 \pm 2.98$ & $143.29 \pm 4.51$ & 0.082 \\
\hline Digestive tract reconstruction time ( $\mathrm{min}$ ) & $53.85 \pm 4.51$ & $50.22 \pm 3.82$ & $31.14 \pm 5.23$ & $<0.01$ \\
\hline Intraoperative blood loss (ml) & $139.09 \pm 8.85$ & $140.22 \pm 7.93$ & $139.78 \pm 8.52$ & 0.634 \\
\hline First anal exhaust time (h) & $59.95 \pm 5.09$ & $60.04 \pm 6.53$ & $60.49 \pm 6.31$ & 0.791 \\
\hline Postoperative hospital stay (d) & $10.53 \pm 1.31$ & $10.52 \pm 1.18$ & $10.68 \pm 1.35$ & 0.636 \\
\hline
\end{tabular}

As shown in Table 6, the differences in body weight, hemoglobin, total serum protein, serum albumin, and blood vitamin B12 between the three groups at 3, 6, 12, and 18 months after surgery were statistically significant $(P<0.05)$. PJIRSTR group had a small fluctuation range of postoperative nutrition indexes and had basically recovered to the preoperative level at 18 months. The nutritional indexes in TGRY group fluctuated a lot postoperatively, especially the levels of hemoglobin and blood vitamin B12, which decreased gradually due to gastrectomy.

\section{Discussion}

For the surgical treatment of stage I and II gastric cancer, currently functional preservation is preferred to reduce long-term postoperative complications and improve the quality of life [10-12]. For patients with gastric cancer, choosing the appropriate method of digestive tract reconstruction directly determines the postoperative quality of life and nutritional status [13-16]. TGRY has been adopted for digestive tract reconstruction for nearly 10 years for Siewert II and III AEG

Table 3 Comparison of postoperative complications among three groups

\begin{tabular}{lllll}
\hline Complications $(n, \%)$ & $\begin{array}{l}\text { PJIRSTR } \\
(n=98)\end{array}$ & $\begin{array}{l}\text { PJIRDTR } \\
(n=103)\end{array}$ & $\begin{array}{l}\text { TGRY } \\
(n=99)\end{array}$ & P value \\
\hline Anastomotic leakage & 0 & $1(0.97)$ & $1(1.01)$ & 0.999 \\
Anastomotic bleeding & 0 & 0 & 0 & \\
Abdominal infection & $1(1.02)$ & 0 & 0 & \\
Incision infection & $1(1.02)$ & $1(0.97)$ & 0 & \\
lleus & 0 & 0 & $1(1.01)$ & \\
Dumping syndrome & 0 & 0 & 0 & \\
\hline
\end{tabular}

patients. However, the development of PG has been limited due to the high incidence of reflux esophagitis and the decline of postoperative quality of life. After continuous improvement and development of surgical procedures, PJIR has become an ideal choice for the treatment of AEG. In addition, other methods including esophagogastrostomy (EG) [6, 17], jejunal interposition (JI) [18, 19], jejunal pouch interposition (JPI) [20, 21], and double tract (DT) $[22,23]$ are also available, among which EG is the most widely used due to simple operation [24]. However, severe reflux esophagitis is often associated with postoperative EG. Therefore, according to the location and size of the tumor, most surgeons currently perform total gastrectomy $[25,26]$, and a few surgeons adopt other reconstruction methods, such as JI, JPI, or DT, to minimize the occurrence of reflux esophagitis and other postoperative complications. In this study, Siewert II or III AEG patients were selected as the research objects to analyze the advantages and disadvantages of three methods of digestive tract reconstruction.

The results of this study showed that there were no significant differences in intraoperative blood loss, anal first exhaust time, and postoperative hospital stay among three groups. Although there were no significant differences in intraoperative and postoperative complications

Table 4 Comparison of the incidence of endoscopic gastroesophageal reflux among three groups

\begin{tabular}{lllll}
\hline Time $(n, \%)$ & $\begin{array}{l}\text { PJIRSTR } \\
(n=98)\end{array}$ & $\begin{array}{l}\text { PJIRDTR } \\
(n=103)\end{array}$ & $\begin{array}{l}\text { TGRY } \\
(n=99)\end{array}$ & $P$ value \\
\hline 3 months & $2(2)$ & $2(1.9)$ & $15(15.2)$ & $<0.01$ \\
6 months & $2(2)$ & $3(2.9)$ & $18(18.2)$ & $<0.01$ \\
12 months & $3(3.1)$ & $3(2.9)$ & $18(18.2)$ & $<0.01$ \\
18 months & $3(3.1)$ & $3(2.9)$ & $20(18.2)$ & $<0.01$ \\
\hline
\end{tabular}


Table 5 Gastroesophageal reflux symptom evaluation (Visick score) for three groups at postoperative 18 months

\begin{tabular}{lllll}
\hline Visick grade $(n, \%)$ & $\begin{array}{l}\text { PJIRSTR } \\
(n=98)\end{array}$ & $\begin{array}{l}\text { PJIRDTR } \\
(n=103)\end{array}$ & $\begin{array}{l}\text { TGRY } \\
(n=99)\end{array}$ & $P$ value \\
\hline I & $94(95.9)$ & $94(91.3)$ & $84(84.8)$ & 0.03 \\
II & $3(3.1)$ & $6(5.9)$ & $7(7.1)$ & 0.44 \\
III & $1(1.0)$ & $3(2.9)$ & $4(4.0)$ & 0.42 \\
IV & 0 & 0 & $4(4.0)$ & 0.02 \\
\hline
\end{tabular}

among the three types of digestive tract reconstruction, for the evaluation of postoperative reflux esophagitis and nutritional indicators, we found that PJIR had significant advantages in improving the nutritional status of patients and reducing the incidence of reflux esophagitis compared with TGRY. We speculated that one of the reasons for the lower incidence of reflux esophagitis of PJIRSTR and PJIRDTR group is that the jejunum interposition has an anti-reflux effect, making reflux fluid unable to flow or only a small amount of reflux into the esophagus, thereby reducing the incidence of reflux esophagitis or reducing reflux symptoms. In addition, the PJIRSTR group blocked the channel of proximal jejunal digestive fluid flowing back into the esophagus, which greatly reduces the occurrence of alkaline reflux esophagitis. Therefore, patients in PJIRSTR group had fewer patients of Visick grade II and III than PJIRDTR group, PJIRDTR group had fewer patients of Visick grade II and III than TGRY group, and TGRY group had patients of Visick grade IV. Nozaki [27] and Namikawa [28] reported that the incidence of reflux esophagitis does not significantly differ between the proximal gastrectomy with jejunal interposition and TGRY. However, in our study, four Visick grade IV patients with severe reflux esophagitis appeared in the TGRY group. We speculated that PJIRDTR provides double output channels for food transit, and this split transit approach can effectively prevent and reduce the incidence of esophageal reflux [29].

The function-preserving operation retained the distal stomach, increased the single-meal food intake, reduced the number of meals, and improved the quality of life [27]. Postoperative nutritional status at postoperative 3,
6, 12, and 18 months including body weight, hemoglobin, total serum protein, serum albumin, and blood vitamin B12 in PJIRSTR group was stable and reached the preoperative level by 18 months. It may be related to the fact that for PJIRSTR group, food can enter into the residual stomach through the interposition jejunum and was fully digested with gastric acid and then entered into the duodenum to stimulate the secretion of various hormones, which is more consistent with the physiological pathway of food. A retrospective study of 1061 cases of total gastrectomy, proximal gastrectomy, or distal gastrectomy in Japan [30] showed that PG had a better prognosis than TG and duodenal pathway reconstruction had a better prognosis than non-duodenal pathway. It is recommended to minimize gastrectomy and retain duodenal food flow pathway without residual cancer in the residual stomach. The decline of each index in PJIRDTR group was larger than that PJIRSTR group whereas was smaller than that in TGRY group. It is possible that most of the food in PJIRDTR group is discharged into the intestinal tract without full digestion, which thereby affects the absorption of food, increases the feeling of abdominal fullness, and reduces the food intake, eventually affecting the patient's nutritional status [31-33]. Furthermore, the remnant gastric antrum provides the capacity for food storage, which not only delays emptying time to ensure the efficient mixing of food with the digestive juices but also promotes gastrin (GAS) secretion for adequate chymus digestion, ultimately enhancing patients' long-term quality of life $[17,29]$. Patients in TGRY group, on the other hand, were in poorer nutritional status than the other two groups because the food enter directly into the intestinal tract and there was no digestive function in the stomach [34]. Jung et al. [35] thought that the change rate of body weight in laparoscopic proximal gastrectomy with double-tract reconstruction (LPG-DT) group was significantly lower than in laparoscopic total gastrectomy (LTG) group. The serum vitamin B12 level in the LPG-DT group was significantly higher than in the LTG group. Research by Kim and his colleagues [36] observed that LPG-DT was beneficial with regard to the absorption of iron and vitamin B12 compared to LTG. A

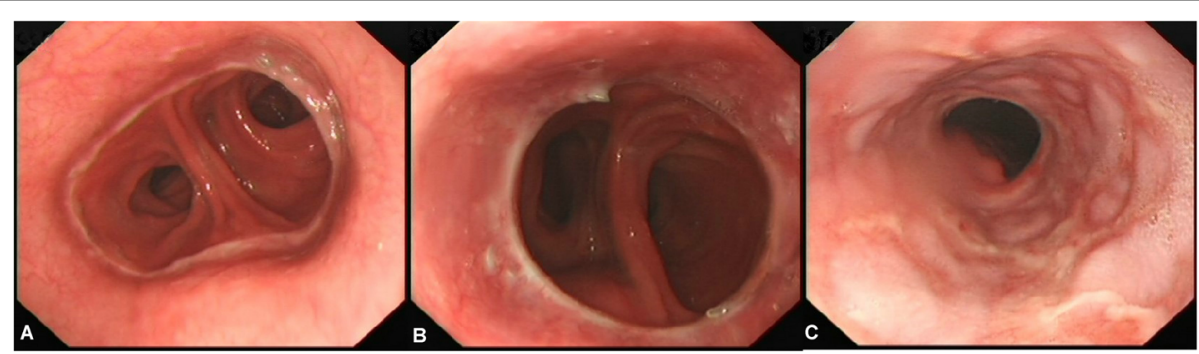

Fig. 3 Gastroscopic examination of anastomotic site after (a) PJIRSTR, (b) PJIRDTR, and (c) TGRY 
Table 6 Comparison of nutrition indexes among three groups

\begin{tabular}{|c|c|c|c|c|}
\hline & $\begin{array}{l}\text { PJIRSTR } \\
(n=98)\end{array}$ & $\begin{array}{l}\text { PJIRDTR } \\
(n=103)\end{array}$ & $\begin{array}{l}\text { TGRY } \\
(n=99)\end{array}$ & $P$ value \\
\hline \multicolumn{5}{|l|}{ Weight } \\
\hline Preoperative & $66.33 \pm 3.116$ & $66.10 \pm 3.618$ & $66.36 \pm 3.824$ & 0.844 \\
\hline Postoperative 3 months & $59.54 \pm 4.393$ & $58.47 \pm 4.385$ & $57.36 \pm 5.683$ & 0.008 \\
\hline Postoperative 6 months & $62.57 \pm 3.407$ & $60.80 \pm 3.851$ & $56.59 \pm 4.936$ & $<0.001$ \\
\hline Postoperative 12 months & $63.17 \pm 3.162$ & $62.27 \pm 3.425$ & $57.84 \pm 4.117$ & $<0.001$ \\
\hline Postoperative 18 months & $64.85 \pm 3.020$ & $64.08 \pm 2.569$ & $58.25 \pm 4.246$ & $<0.001$ \\
\hline \multicolumn{5}{|l|}{ Hemoglobin } \\
\hline Preoperative & $126.64 \pm 5.562$ & $128.06 \pm 4.671$ & $127.00 \pm 5.449$ & 0.137 \\
\hline Postoperative 3 months & $120.24 \pm 4.393$ & $119.29 \pm 4.807$ & $114.48 \pm 4.767$ & $<0.001$ \\
\hline Postoperative 6 months & $118.27 \pm 4.123$ & $117.40 \pm 4.138$ & $111.11 \pm 5.099$ & $<0.001$ \\
\hline Postoperative 12 months & $120.98 \pm 3.287$ & $120.59 \pm 3.382$ & $115.72 \pm 3.201$ & $<0.001$ \\
\hline Postoperative 18 months & $121.08 \pm 3.289$ & $120.56 \pm 2.404$ & $118.83 \pm 3.207$ & $<0.001$ \\
\hline \multicolumn{5}{|l|}{ Total serum protein } \\
\hline Preoperative & $72.12 \pm 3.967$ & $72.60 \pm 3.861$ & $72.69 \pm 3.762$ & 0.543 \\
\hline Postoperative 3 months & $71.04 \pm 3.548$ & $71.40 \pm 2.684$ & $69.39 \pm 2.494$ & $<0.001$ \\
\hline Postoperative 6 months & $68.36 \pm 3.253$ & $68.46 \pm 3.183$ & $65.34 \pm 2.177$ & $<0.001$ \\
\hline Postoperative 12 months & $71.73 \pm 2.595$ & $70.73 \pm 3.075$ & $68.74 \pm 1.747$ & $<0.001$ \\
\hline Postoperative 18 months & $71.45 \pm 1.916$ & $71.54 \pm 1.984$ & $70.09 \pm 1.660$ & $<0.001$ \\
\hline \multicolumn{5}{|l|}{ Serum albumin } \\
\hline Preoperative & $46.07 \pm 2.412$ & $45.50 \pm 2.807$ & $46.21 \pm 3.354$ & 0.183 \\
\hline Postoperative 3 months & $44.09 \pm 1.777$ & $42.48 \pm 2.244$ & $42.63 \pm 2.068$ & $<0.001$ \\
\hline Postoperative 6 months & $44.38 \pm 1.897$ & $43.81 \pm 1.966$ & $41.00 \pm 1.922$ & $<0.001$ \\
\hline Postoperative 12 months & $44.76 \pm 1.878$ & $44.60 \pm 2.157$ & $42.84 \pm 2.315$ & $<0.001$ \\
\hline Postoperative 18 months & $46.27 \pm 2.952$ & $44.00 \pm 2.240$ & $43.13 \pm 2.207$ & $<0.001$ \\
\hline \multicolumn{5}{|l|}{ Vitamin 12} \\
\hline Preoperative & $177.16 \pm 5.807$ & $176.05 \pm 3.889$ & $176.04 \pm 5.443$ & 0.248 \\
\hline Postoperative 3 months & $170.26 \pm 3.862$ & $168.08 \pm 3.741$ & $157.02 \pm 4.679$ & $<0.001$ \\
\hline Postoperative 6 months & $164.98 \pm 3.956$ & $154.13 \pm 6.981$ & $139.19 \pm 4.690$ & $<0.001$ \\
\hline Postoperative 12 months & $167.87 \pm 2.987$ & $144.77 \pm 6.588$ & $114.86 \pm 9.765$ & $<0.001$ \\
\hline Postoperative 18 months & $164.56 \pm 3.840$ & $147.16 \pm 6.624$ & $104.36 \pm 9.494$ & $<0.001$ \\
\hline
\end{tabular}

recent study has shown that body weight and skeletal muscle index reduction rates were lower in the LPG-DT group than in the LTG group [37]. Although distal stomach was retained in PJIRDTR group and PJIRSTR group and gastric mucosa could continue to produce internal factors for hematopoiesis, postoperative follow-up data showed a downward trend or even mild anemia of hemoglobin and vitamin B12 in the two groups, which may be related to the decrease in the number of gastric wall cells and changes in the acid and alkali environment in the stomach. VB12 deficiency, which causes megaloblastic anemia and a spectrum of neuropsychiatric disorders, is one of the common long-term nutritional sequelae after gastrectomy [35, 38]. The acidic environment of the stomach facilitates the breakdown of vitamin B12 that is bound to food. Intrinsic factor, which is released by parietal cells in the stomach, binds to vitamin B12 in the duodenum. This vitamin B12-intrinsic factor complex subsequently aids in the absorption of vitamin B12 in the terminal ileum [35]. Detailedly, the alkaline digestive fluid from jejunum in PJIRDTR group flows back into the residual stomach, causing the increase of $\mathrm{PH}$ value in the residual stomach and the production obstacle of internal factor. Therefore, lack of internal factor, B12, or tetrahydrofolate can cause the development of the nucleus to lag behind the development of the cytoplasm and eventually resulting in megaloblastic anemia [24].

Loss of the normal physiological function in TGRY group, due to the excision of the distal residual stomach, 
can make patients more prone to malnutrition and lose weight postoperatively. For PJIRDTR group and PJIRSTR group, retention of the distal stomach maintains the normal gastrointestinal anatomy and part of the physiological functions, which is beneficial to the digestion and absorption of postoperative nutrition and can also improve the postoperative tolerance of chemotherapy. Takiguchi et al. [39] evaluated subjective symptoms using a well-designed validated questionnaire and a post-gastrectomy syndrome assessment scale (PGSAS45). Their data showed that PG was significantly improved over TG in terms of preventing body weight loss, the necessity for additional meals, diarrhea, and dumping.

The limitation of this study is that the survival rate of the patients was not statistically analyzed in this study, which needs to be further studied.

In summary, these three methods of digestive tract reconstruction are safe. PJIR is suitable for AEG patients of Siewert II and III. PJIRSTR is preferred since it had good anti-reflux effect, improved the long-term nutritional status and living quality, and the operation was not complicated. Multi-center and long-term follow-up studies remain to be done to clarify this finding.

\section{Abbreviations}

AEG: Adenocarcinoma of esophagogastric junction; DT: Double tract: EG: Esophagogastrostomy; J: Jejunal interposition; JPI: Jejunal pouch interposition; PG: Proximal gastrectomy; PJIR: Piggyback jejunal interposition reconstruction; PJIRDTR: Piggyback jejunal interposition reconstruction double-tract reconstruction; PJIRSTR: Piggyback jejunal interposition reconstruction single-tract reconstruction; TGRY: Total gastrectomy esophageal jejunal Roux-en-Y anastomosis

\section{Acknowledgements}

Not applicable.

\section{Authors' contributions}

ZL and JD conceived the study. ZL performed the literature search and writing of the manuscript. ZL analyzed and interpreted the data. $\mathrm{QH}, \mathrm{WZ}$, and KT collected and assembled the data. JD submitted the manuscript and is the corresponding author. All authors read and approved the final manuscript.

\section{Funding}

This study was supported by Key Research and Development (R\&D) Projects of Shanxi Province (no.201803D31167 and no.20150313007-7).

\section{Availability of data and materials}

The data used to support the findings of this study are available from the corresponding author upon request.

\section{Ethics approval and consent to participate}

This study was approved by the ethics committee of Shanxi Tumor Hospital. All patients signed informed consent.

\section{Consent for publication}

The patient was given his consent for information about himself to be published in World Journal of Surgical Oncology.

\section{Competing interests}

The authors declare that they have no competing interests.
Received: 6 September 2019 Accepted: 26 November 2019

Published online: 06 December 2019

\section{References}

1. Goto H, Tokunaga M, Miki Y, Makuuchi R, Sugisawa N, Tanizawa Y, Bando E, Kawamura T, Niihara M, Tsubosa $Y$, Terashima M. The optimal extent of lymph node dissection for adenocarcinoma of the esophagogastric junction differs between Siewert type II and Siewert type III patients. Gastric Cancer. 2014

2. Kurokawa Y, Hiki N, Yoshikawa T, Kishi K, Ito Y, Ohi M, Wada N, Takiguchi S, Mine S, Hasegawa S, Matsuda T, Takeuchi H. Mediastinal lymph node metastasis and recurrence in adenocarcinoma of the esophagogastric junction. Surgery. 2015;157:551-5.

3. Wu AJ, Bosch WR, Chang DT, Hong TS, Jabbour SK, Kleinberg LR, Mamon HJ, Thomas CR Jr, Goodman KA. Expert consensus contouring guidelines for intensity modulated radiation therapy in esophageal and gastroesophageal junction cancer. Int J Radiat Oncol Biol Phys. 2015;92:911-20.

4. Rosa F, Quero G, Fiorillo C, Bissolati M, Cipollari C, Rausei S, Chiari D, Ruspi L, de Manzoni G, Costamagna G, Doglietto GB, Alfieri S. Total vs proximal gastrectomy for adenocarcinoma of the upper third of the stomach: a propensity-score-matched analysis of a multicenter western experience (On behalf of the Italian Research Group for Gastric Cancer-GIRCG). Gastric Cancer. 2018;21:845-52.

5. Bogoevski D, Bockhorn M, Koenig A, Reeh M, von Loga K, Sauter G, Rosch T, Izbicki JR. How radical should surgery be for early esophageal cancer? World J Surg. 2011:35:1311-20.

6. Harrison LE, Karpeh MS, Brennan MF. Total gastrectomy is not necessary for proximal gastric cancer. Surgery. 1998;123:127-30.

7. Pu YW, Gong W, Wu YY, Chen Q, He TF, Xing CG. Proximal gastrectomy versus total gastrectomy for proximal gastric carcinoma. A meta-analysis on postoperative complications, 5-year survival, and recurrence rate. Saudi Med J. 2013;34:1223-8.

8. Tao K, Dong JH. Phase I clinical research of jejunal interposition in adenocarcinoma of the esophagogastric junction II/III proximal gastrectomy. Gastroenterol Res Pract. 2016;2016:1639654.

9. Japanese Classification of Esophageal Cancer, 11th Edition: part I. Esophagus 2017;14:1-36.

10. Hiki N, Nunobe S, Kubota T, Jiang X. Function-preserving gastrectomy for early gastric cancer. Ann Surg Oncol. 2013;20:2683-92.

11. Koeda K, Nishizuka S, Wakabayashi G. Minimally invasive surgery for gastric cancer: the future standard of care. World J Surg. 2011;35:1469-77.

12. Takayama T, Matsumoto S, Wakatsuki K, Tanaka T, Migita K, Ito M, Nakajima $Y$. Novel laparoscopic procedure for treating proximal early gastric cancer: laparoscopy-assisted pylorus-preserving nearly total gastrectomy. Surg Today. 2014:44:2332-8

13. Chin AC, Espat NJ. Total gastrectomy: options for the restoration of gastrointestinal continuity. Lancet Oncol. 2003;4:271-6.

14. Hosogi H, Yoshimura F, Yamaura T, Satoh S, Uyama I, Kanaya S. Esophagogastric tube reconstruction with stapled pseudo-fornix in laparoscopic proximal gastrectomy: a novel technique proposed for Siewert type II tumors. Langenbecks Arch Surg. 2014;399:517-23.

15. LaFemina J, Vinuela EF, Schattner MA, Gerdes H, Strong VE. Esophagojejunal reconstruction after total gastrectomy for gastric cancer using a transorally inserted anvil delivery system. Ann Surg Oncol. 2013;20:2975-83.

16. Ichikawa D, Komatsu S, Kubota T, Okamoto K, Shiozaki A, Fujiwara H, Otsuji E. Long-term outcomes of patients who underwent limited proximal gastrectomy. Gastric Cancer. 2014;17:141-5.

17. Shiraishi N, Adachi Y, Kitano S, Kakisako K, Inomata M, Yasuda K. Clinical outcome of proximal versus total gastrectomy for proximal gastric cancer. World J Surg. 2002;26:1150-4.

18. Katai H, Sano T, Fukagawa T, Shinohara H, Sasako M. Prospective study of proximal gastrectomy for early gastric cancer in the upper third of the stomach. Br J Surg. 2003;90:850-3.

19. Katai H, Morita S, Saka M, Taniguchi H, Fukagawa T. Long-term outcome after proximal gastrectomy with jejunal interposition for suspected early cancer in the upper third of the stomach. Br J Surg. 2010;97:558-62.

20. Takagawa R, Kunisaki C, Kimura J, Makino H, Kosaka T, Ono HA, Akiyama H, Endo I. A pilot study comparing jejunal pouch and jejunal interposition reconstruction after proximal gastrectomy. Dig Surg. 2010;27:502-8.

21. Iwata T, Kurita $N$, Ikemoto $T$, Nishioka M, Andoh T, Shimada M. Evaluation of reconstruction after proximal gastrectomy: prospective comparative study 
of jejunal interposition and jejunal pouch interposition Hepatogastroenterology. 2006;53:301-3.

22. Jung DH, Lee Y, Kim DW, Park YS, Ahn SH, Park DJ, Kim HH. Laparoscopic proximal gastrectomy with double tract reconstruction is superior to laparoscopic total gastrectomy for proximal early gastric cancer. Surg Endosc. 2017;31:3961-9.

23. Nomura E, Lee SW, Kawai M, Yamazaki M, Nabeshima K, Nakamura K, Uchiyama K. Functional outcomes by reconstruction technique following laparoscopic proximal gastrectomy for gastric cancer: double tract versus jejunal interposition. World J Surg Oncol. 2014;12:20.

24. Ichikawa D, Ueshima Y, Shirono K, Kan K, Shioaki Y, Lee CJ, Hamashima T, Deguchi E, Ikeda E, Mutoh F, Oka T, Kurioka H. Esophagogastrostomy reconstruction after limited proximal gastrectomy. Hepatogastroenterology. 2001;48:1797-801.

25. An JY, Youn HG, Choi MG, Noh JH, Sohn TS, Kim S. The difficult choice between total and proximal gastrectomy in proximal early gastric cancer. Am J Surg. 2008;196:587-91.

26. Tokunaga M, Ohyama S, Hiki N, Hoshino E, Nunobe S, Fukunaga T, Seto Y, Yamaguchi T. Endoscopic evaluation of reflux esophagitis after proximal gastrectomy: comparison between esophagogastric anastomosis and jejunal interposition. World J Surg. 2008:32:1473-7.

27. Nozaki I, Hato S, Kobatake T, Ohta K, Kubo Y, Kurita A. Long-term outcome after proximal gastrectomy with jejunal interposition for gastric cancer compared with total gastrectomy. World J Surg. 2013;37:558-64.

28. Namikawa T, Oki T, Kitagawa H, Okabayashi T, Kobayashi M, Hanazaki K. Impact of jejunal pouch interposition reconstruction after proximal gastrectomy for early gastric cancer on quality of life: short- and long-term consequences. Am J Surg. 2012;204:203-9.

29. Xiao JW, Liu ZL, Ye PC, Luo YJ, Fu ZM, Zou Q, Wei SJ. Clinical comparison of antrum-preserving double tract reconstruction vs roux-en-Y reconstruction after gastrectomy for Siewert types $I$ and III adenocarcinoma of the esophagogastric junction. World J Gastroenterol. 2015;21:9999-10007.

30. Ogoshi K, Okamoto Y, Nabeshima K, Morita M, Nakamura K, Iwata K, Soeda J, Kondoh Y, Makuuchi H. Focus on the conditions of resection and reconstruction in gastric cancer. What extent of resection and what kind of reconstruction provide the best outcomes for gastric cancer patients? Digestion. 2005;71:213-24.

31. Tanaka K, Ebihara Y, Kurashima Y, Nakanishi Y, Asano T, Noji T, Murakami S, Nakamura T, Tsuchikawa T, Okamura K, Shichinohe T, Hirano S. Laparoscopic proximal gastrectomy with oblique jejunogastrostomy. Langenbecks Arch Surg. 2017;402:995-1002.

32. Aikou T, Natsugoe S, Shimazu H, Nishi M. Antrum preserving double tract method for reconstruction following proximal gastrectomy. Jpn J Surg. 1988:18:114-5.

33. Hosoda K, Yamashita K, Moriya H, Washio M, Mieno H, Ema A, Watanabe M. Esophagogastric junction cancer successfully treated by laparoscopic proximal gastrectomy and lower esophagectomy with intrathoracic doubleflap technique: A case report. Asian J Endosc Surg. 2018;11:160-4

34. Olbe L, Lundell L. Intestinal function after total gastrectomy and possible consequences of gastric replacement. World J Surg. 1987;11:713-9.

35. Oh R, Brown DL. Vitamin B12 deficiency. Am Fam Physician. 2003;67:979-86.

36. Kim DJ, Kim W. Laparoscopy-assisted proximal gastrectomy with double tract anastomosis is beneficial for vitamin B12 and iron absorption. Anticancer Res. 2016;36:4753-8.

37. Sugiyama M, Oki E, Ando K, Nakashima Y, Saeki H, Maehara Y. Laparoscopic proximal gastrectomy maintains body weight and skeletal muscle better than total gastrectomy. World J Surg. 2018:42:3270-6.

38. Fernandez-Banares F, Monzon H, Forne M. A short review of malabsorption and anemia. World I Gastroenterol. 2009:15:4644-52

39. Takiguchi N, Takahashi M, Ikeda M, Inagawa S, Ueda S, Nobuoka T, Ota M, Iwasaki Y, Uchida N, Kodera Y, Nakada K. Long-term quality-of-life comparison of total gastrectomy and proximal gastrectomy by postgastrectomy syndrome assessment scale (PGSAS-45): a nationwide multi-institutional study. Gastric Cancer. 2015;18:407-16.

\section{Publisher's Note}

Springer Nature remains neutral with regard to jurisdictional claims in published maps and institutional affiliations.

\section{Ready to submit your research? Choose BMC and benefit from:}

- fast, convenient online submission

- thorough peer review by experienced researchers in your field

- rapid publication on acceptance

- support for research data, including large and complex data types

- gold Open Access which fosters wider collaboration and increased citations

- maximum visibility for your research: over $100 \mathrm{M}$ website views per year

At BMC, research is always in progress.

Learn more biomedcentral.com/submissions 